\title{
Development and Verification of Unstructured Adaptive Mesh Technique with Edge Compatibility*
}

\author{
Kei ITO $^{* *}$, Tomoaki KUNUGI ${ }^{* * *}$ and Hiroyuki OHSHIMA* ${ }^{* *}$ \\ **Advanced Nuclear System Research and Development Directorate, Japan Atomic Energy Agency, \\ 4002 Narita, O-arai-machi, Ibaraki, 311-1393, Japan \\ E-mail: ito.kei@jaea.go.jp

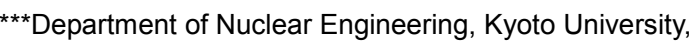 \\ Yoshida-honmachi, Sakyo-ku, Kyoto-shi, Kyoto, 606-8501, Japan
}

\begin{abstract}
In the design study of the large-sized sodium-cooled fast reactor (JSFR), one key issue is suppression of gas entrainment (GE) phenomena at a gas-liquid interface. Therefore, the authors have been developed a high-precision CFD algorithm to evaluate the GE phenomena accurately. The CFD algorithm has been developed on unstructured meshes to establish an accurate modeling of JSFR system. For two-phase interfacial flow simulations, a high-precision volume-of-fluid algorithm is employed. It was confirmed that the developed CFD algorithm could reproduce the GE phenomena in a simple GE experiment. Recently, the authors have been developed an important technique for the simulation of the GE phenomena in JSFR. That is an unstructured adaptive mesh technique which can apply fine cells dynamically to the region where the GE occurs in JSFR. In this paper, as a part of the development, a two-dimensional unstructured adaptive mesh technique is discussed. In the two-dimensional adaptive mesh technique, each cell is refined isotropically to reduce distortions of the mesh. In addition, connection cells are formed to eliminate the edge incompatibility between refined and non-refined cells. The two-dimensional unstructured adaptive mesh technique is verified by solving well-known lid-driven cavity flow problem. As a result, the two-dimensional unstructured adaptive mesh technique succeeds in providing a high-precision solution, even though poor-quality distorted initial mesh is employed. In addition, the simulation error on the two-dimensional unstructured adaptive mesh is much less than the error on the structured mesh with a larger number of cells.
\end{abstract}

Key words: Numerical Simulation, Gas-Liquid Two-Phase Flow, Interfacial Flow, Volume of Fluid, Unstructured Adaptive Mesh, Gas Entrainment

\section{Introduction}

Fast breeder reactors are located as one of the possible energy sources in the future. In Japan, the fast reactor cycle technology development project has been conducted by Japan Atomic Energy Agency and related organizations to establish an economically superior design of the large-sized sodium-cooled fast reactor (JSFR) ${ }^{(1)}$. In the design study, one key issue is suppression of a GE phenomena at a gas-liquid interface in the reactor vessel because larger coolant velocity in the JSFR than conventional designs can induce interfacial fluctuation and resulting GE occurrences at upper plenum region in the reactor vessel. Since bubbles entrained to the coolant due to the GE phenomena might cause power disturbance 
when they go through the reactor core, the GE phenomena should be suppressed in the JSFR for a stable operation.

A GE phenomena have been studied experimentally and theoretically in many years ${ }^{(2)}$. In the studies, onset conditions of the GE phenomena were investigated using experimental systems consisting of reservoir tanks or main pipes with branches. From the experimental results, the correlation of the onset conditions were formed as an equation of Froude number (Fr) and branch diameter ${ }^{(3)}$. Also for FBR systems, some studies were conducted and the GE phenomena in the FBR systems were classified into three types, i.e. waterfall, interfacial disturbance and vortical flow types ${ }^{(4)}$. Among them, former two types can be suppressed by reducing horizontal velocity at the gas-liquid interface in the reactor vessel under a certain level (about $0.10 \mathrm{~m} / \mathrm{s}$ ). On the other hand, the GE phenomena induced by vortical flows are very difficult to suppress by a universal rule. Since vortical flows at the gas-liquid interface in the reactor vessel are formed locally and transiently, it is evident that a lot of parameters, e.g. local system configurations, are related to vortical flow properties. Therefore, the authors are focusing on the evaluation of the vortical flow type GE phenomena in the GE study.

As the first step of the GE study, a CFD-based prediction method for occurrences of the vortical flow type GE phenomena was constructed ${ }^{(5)}$. This prediction method determines vortical flow regions from a transient CFD result on a relatively coarse mesh without considering interfacial deformations. However, this prediction method cannot evaluate vortical flow properties, e.g. circulations of each vortex, directly from the CFD result due to the lack of mesh resolution. Therefore, a vortical flow model was constructed based on the famous Burgers model ${ }^{(6)}$ and applied to the CFD result to calculate circulations, downward velocity gradients and gas core lengths of each vortex. Finally, the GE occurrences for each vortex can be judged by comparing calculated values to an onset condition maps for vortical flow type GE phenomena. For this comparison, two onset condition maps were organized based on experimental data for a gas core length and a downward velocity gradient, respectively. This procedure was validated by predicting onset conditions of the GE in the 1/1.8-scaled partial upper plenum model of the $\mathrm{JSFR}^{(7)}$. As a result, the experimental onset conditions could be evaluated properly by the prediction method.

Though the prediction method is very useful and effective for the design study of the JSFR because CFD on relatively coarse mesh can be conducted within practical simulation time, due to the lack of considering interfacial deformations, the amount of entrained gas is not evaluated by the prediction method. If an amount of entrained gas by the GE phenomena is evaluated accurately, it is very useful to optimize the design of the JSFR. Therefore, as the second step of the GE study, the authors are developing the evaluation method for an amount of entrained gas based on a CFD algorithm. In the previous study ${ }^{(8)}$, the authors presented the applicability of a commercial CFD code to the vortical flow type GE phenomena. As a result of the commercial CFD on the simple GE experiment ${ }^{(9)}$, it was confirmed that the vortical flow type GE phenomena occurred in the commercial CFD result but the commercial CFD code underestimates the onset condition of the GE phenomena compared to the experimental data. Therefore, the authors are developing a high-precision CFD algorithm for gas-liquid two-phase flows to evaluate the vortical flow type GE phenomena accurately ${ }^{(10)}$. In this development, unstructured meshes were employed to establish accurate geometrical modeling of complicated JSFR systems because accurate geometrical modeling is important to simulate complicated flow patterns accurately in the JSFR due to high dependency of vortical flows on local system configurations. As a gas-liquid two-phase flow simulation algorithm, a volume-tracking algorithm based on a high-precision volume-of-fluid algorithm, namely PLIC (Piecewise Linear Interface Calculation ${ }^{(11)}$ ), was employed and newly developed on fully unstructured meshes based on the conventional algorithms on structured meshes ${ }^{(12)}$. In addition, the 
authors developed a new volume-conservative algorithm establishing perfect volume conservation for each phase (gas and liquid phases). New algorithms were introduced also to velocity and pressure calculation procedures to eliminate unphysical behaviors induced by the mechanical unbalance between pressure and surface tension forces ${ }^{(13)}$. Finally, the CFD algorithm was validated by simulating the simple GE experiment and succeeded in reproducing the experimental onset condition of the vortical flow type GE phenomena ${ }^{(10)}$. Here, it should be noted that, to obtain the accurate simulation result for the vortical flow type GE phenomena, very fine cells have to be applied to vortical flow regions. This constrain is not severe in the numerical simulations of the simple GE experiment where only one vortical flow region exists. However, in the numerical simulations of the upper plenum region of JSFR, the constraint becomes a critical issue because it is too difficult to predict vortical flow regions in the region. In this case, there are two ways to simulate the GE phenomena in JSFR. One is an iterative simulation where preliminary simulations are conducted to obtain appropriate mesh arrangement for the true simulation. This way is convenient because no improvement is necessary on the CFD algorithm but is so time consuming. The other way is the employment of an adaptive mesh technique. By using an adaptive mesh technique, dynamic mesh redistribution is achieved and cells in vortical flow regions can be refined automatically. Therefore, the authors decided to develop an adaptive mesh technique to simulate the GE phenomena in JSFR.

Adaptive mesh techniques for CFD algorithms have been studied widely, especially on aerospace field. A lot of useful techniques are proposed and among them, the bi-section ${ }^{(14)}$ and Delaunay triangulation ${ }^{(15)}$ methods are especially famous and useful. In the bi-section method, a triangular cell is refined into two triangular cells by the edge connecting one vertex on the original triangular cell and the central point of the facing edge to the vertex. On the other hand, in the Delaunay triangulation method, an arbitrary shape region is refined by high-quality triangular cells. They are useful techniques because they can reduce degradations of mesh quality which occurs commonly when an adaptive mesh technique is applied repeatedly. A local smoothing ${ }^{(16)}$ and an edge (face) swapping ${ }^{(17)}$ methods are also useful to maintain mesh quality. Furthermore, a moving mesh method ${ }^{(18)}$ is often employed. However, these methods are targeting triangular cells (in two-dimensional meshes) or tetrahedral cells (in three-dimensional meshes). Therefore, it is difficult to modify them straightforward to quadrilateral cells (in two-dimensional meshes) and/or prism and hexahedral cells (in three-dimensional meshes) employed in the authors' CFD algorithm. In addition, the high-precision volume-of-fluid algorithm employed in the CFD algorithm is not suited for these famous methods because very complicated geometrical calculations are necessary to redistribute regions occupied by each phase fluid from original cells to refined cells. Though the geometrical calculations are complicated enough even in two-dimensional unstructured meshes, it can be too difficult to be conducted in three-dimensional unstructured meshes. Therefore, the authors must develop a new unstructured adaptive mesh technique suited for the CFD algorithm.

(a)

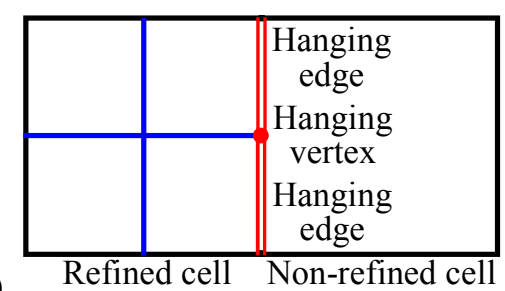

Fig. 1 Comparison of adaptive mesh refinement techniques: (a) edge incompatible structured adaptive mesh, (b) edge compatible unstructured adaptive mesh 
In this paper, as a part of the development of a new unstructured adaptive mesh technique, a two-dimensional unstructured adaptive mesh technique for single-phase flows is developed. Considering the suitability for the high-precision volume-of-fluid algorithm in the authors' CFD algorithm, an isotropic cell refinement method ${ }^{(19,20)}$ is employed. The isotropic cell refinement method is useful because mesh quality is maintained though adaptation procedures. On the other hand, a more general method ${ }^{(21,22)}$ causes degradation of mesh quality because distorted cells are generated. A remaining issue on the isotropic cell refinement method is edge compatibility. In adaptive structured meshes, there can be more than two cells on one edge though only two cells are on usual edges (see Fig. 1(a)). In that case, the edge compatibility is not satisfied and a special treatment is necessary on the incompatible edges. Therefore, due to the edge incompatibility, numerical simulation algorithms near the incompatible edges become complicated. When the isotropic cell refinement method is employed alone, the edge compatibility is not satisfied even on unstructured meshes ${ }^{(23)}$. However, arbitrary mesh arrangements can be conducted on unstructured meshes, and therefore, the edge compatibility can be satisfied by utilizing this characteristic (see Fig. 1(b)). To achieve an edge compatible mesh refinement, the authors introduce connection cells between refined and normal (non-refined) cells. The connection cells eliminate the edge incompatibility and prevent numerical simulation algorithms from complication. In other words, the same numerical simulation algorithms for velocity, pressure or volume-of-fluid calculations can be utilized on both adaptive and non-adaptive meshes. Finally, the developed two-dimensional unstructured adaptive mesh technique for single-phase flows is validated by solving the lid-driven square cavity flow problem to show that the unstructured adaptive mesh technique is useful to simulate vortical flows more accurately and effectively (less number of cells and/or less computational time) compared to a CFD on a non-adaptive mesh.

\section{Two-dimensional Unstructured Adaptive Mesh Technique}

\subsection{Brief Description of Two-dimensional Unstructured Adaptive Mesh Technique}

In the developed two-dimensional unstructured adaptive mesh, triangular and quadrilateral cells with arbitrary shapes are available. In general, these two types of cells are enough to generate simulation meshes for any complicated two-dimensional system configurations. Furthermore, there is no hanging edge and vertex in the unstructured adaptive mesh, which usually exist in a structured adaptive mesh (see Fig. 1(a)), because the authors introduce connection cells between refined and normal (non-refined) cells to eliminate hanging edges and vertices (see Fig. 1(b)). Therefore, the unstructured adaptive mesh technique always satisfies the edge compatibility and does not need any special treatment for flow simulation algorithms on the edges on refined cells. Subdivision patterns of connection cells are shown later in section 2.3. It should be noted that the authors call small cells generated in an original cell "child cells" of the original cell called "parent cell".

Each operation in the two-dimensional unstructured adaptive mesh technique (cell refinement, mergence and introduction of connection cells) is conducted as follows:

1) all connection cells are reduced (child cells in each connection cell are merged into their parent cell);

2) indication values for cell refinement or mergence are calculated for active cells (having no child cell);

3) Refinement or mergence flags are provided if the indication values of each active cell meet refinement or mergence conditions, respectively;

4) If every child cell in a particular parent cell has the mergence flag, the mergence operation is conducted; 
5) Every cells having the refinement flag are refined into four child cells;

6) Connection cells are introduced to satisfy the edge compatibility.

\subsection{Isotropic Cell Refinement Method}

The isotropic cell refinement method is a kind of various cell refinement methods for unstructured cells ${ }^{(19,20)}$. However, the isotropic cell refinement method has several superior characteristics compared to other cell refinement methods. Most importantly, the isotropic cell refinement method is efficient to reduce the degradation of mesh quality from the original mesh when the mesh adaptation is repeatedly applied. Namely, if a more general cell refinement method is employed, it is inevitable that distorted cells are generated and the mesh quality highly decreases when the distorted cells are refined again into further distorted child cells. In addition, the isotropic cell refinement method do not change initial mesh arrangement, namely, child cells are created in their present cell, and initial cells does not changes their shapes. This characteristic is very important for the gas-liquid two-phase flow simulation by the high-precision volume-of-fluid algorithm because the geometrical redistributions of each phase's fluid from a parent cell to its child cells are conducted easily within each parent cell. Therefore, the isotropic cell refinement method seems most suitable to the authors' CFD algorithm.

By applying the isotropic cell refinement method, a triangular cell is refined into four triangular child cells by three new edges joining the central points of three original edges on the parent triangular cell (see refined cell in Fig. 2(a)) and a quadrilateral cell is refined also into four quadrilateral child cells by two new edges joining the central points of original face-to-face edges on the parent quadrilateral cell (see refined cell in Fig. 1(b)).

In single-phase flow simulations, velocity and momentum defined in a parent cell have to be redistributed to its child cells. In the two-dimensional unstructured adaptive mesh technique, the redistributions are calculated by following equations:

$$
\begin{aligned}
& \vec{v}_{\text {child }}=\vec{v}_{\text {prent }}+(\nabla v)_{\text {parent }} \cdot\left(\vec{r}_{\text {child }}-\vec{r}_{\text {parent }}\right), \\
& \vec{m}_{\text {child }}=\rho \vec{v}_{\text {child }}
\end{aligned}
$$

where $\rho, \vec{v},(\nabla v)$ and $\vec{m}$ are the density, velocity vector, velocity gradient tensor and momentum vector, respectively. $\vec{r}$ indicates the position of a cell center. The subscripts child and parent show child and parent cells. It should be noted that Eqs. (1) and (2) can be extended to the gas-liquid two-phase flow by using the volume-weighted centers of gas and liquid phases ${ }^{(24)}$. By summarizing Eq. (1) for every child cell in a parent cell, it can be confirmed that the redistribution method conserve the momentum because $\sum \vec{r}_{\text {child }}=\vec{r}_{\text {parent }}$ is guaranteed by the isotropic cell refinement method. In the cell mergence procedure, the velocity and momentum in a parent cell are calculated in the similar manner.

\subsection{Generation of Connection Cell}

Connection cells are generated between refined and non-refined cells to satisfy the edge compatibility. When a connection cell is generated, an inner subdivision pattern is determined based on the statuses of the edges on the connection cell. Namely, every edge is judged if it is subdivided into two edges or not and this information is used to determine a subdivision pattern. For triangular connection cells, there are three subdivision patterns as shown in Fig. 2(a). On the other hand, quadrilateral connection cells have five subdivision patterns as shown in Fig. 2(b). In the figure, central cells surrounded by red double-lines are the connection cells. It should be noted that a connection cell are consistent with a refinement cell when every edge on the connection cell is subdivided. 
As for the redistribution of the velocity and momentum in a connection cell, the similar redistribution method with that for the cell refinement can be applicable. However, in the connection cell, $\sum \vec{r}_{\text {child }}$ is not always same as $\vec{r}_{\text {parent }}$. Therefore, Eq. (1) is modified to conserve the momentum during the generation of connection cells.

\subsection{Rules for Cell Refinement and Mergence}

The second invariant of velocity gradient tensors, vorticities or magnitudes of velocity gradients in each cell is calculated as the indication values to determine which cell should be refined or merged. For example, when the first indication value (second invariant of velocity gradient tensor) is employed, refined cells are applied efficiently to vortical flow regions. If the indication value of certain cell is larger than the refinement condition, the cell is refined into four child cells. On the other hand, if indication values of every child cell in a particular parent cell are smaller than the mergence condition, the child cells are merged into the parent cell.

A refinement level is set for every cell to specify statuses of the cells. The refinement level changes by the refinement and mergence of cells. By the cell refinement operation, the child cells got the refinement level one level higher than the refinement level of their parent cell. Similarly, the refinement level of a merged cell is one level lower than its child cells. In addition, the range of the refinement level is limited between zero and maximum level which is determined for each simulation case. Usually, the maximum level is set between three and ten. This constraint on the refinement level means that the cell having the maximum level is not refined anymore and initial cells (refinement level is zero) is not merged. In the case of generating connection cells, the child cells in a connection cell have the same refinement level as their parent cell. This rule is necessary to distinguish connection cells from refinement cells.

(a)
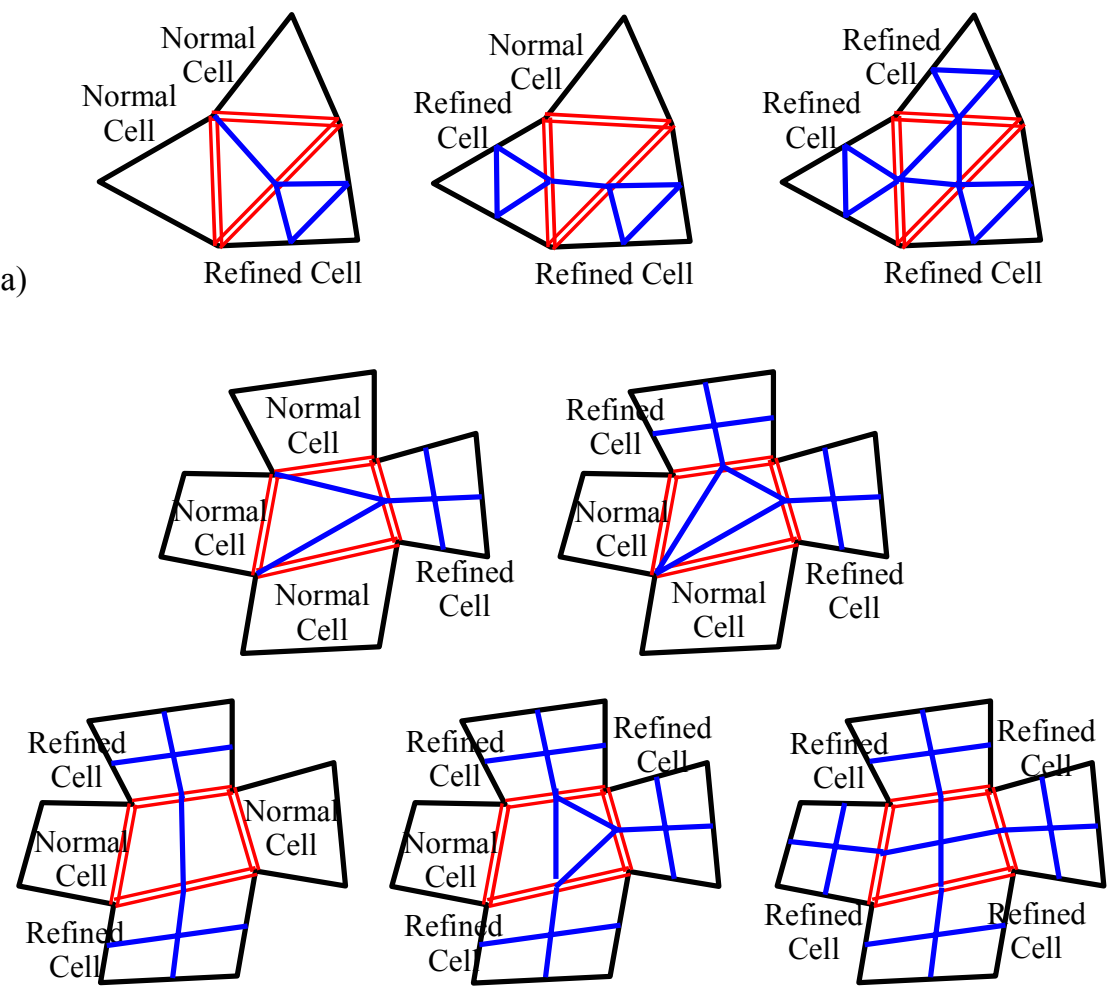

Fig. 2 Subdivision patterns of connection cells: (a) triangular connection cell, (b) quadrilateral connection cell 


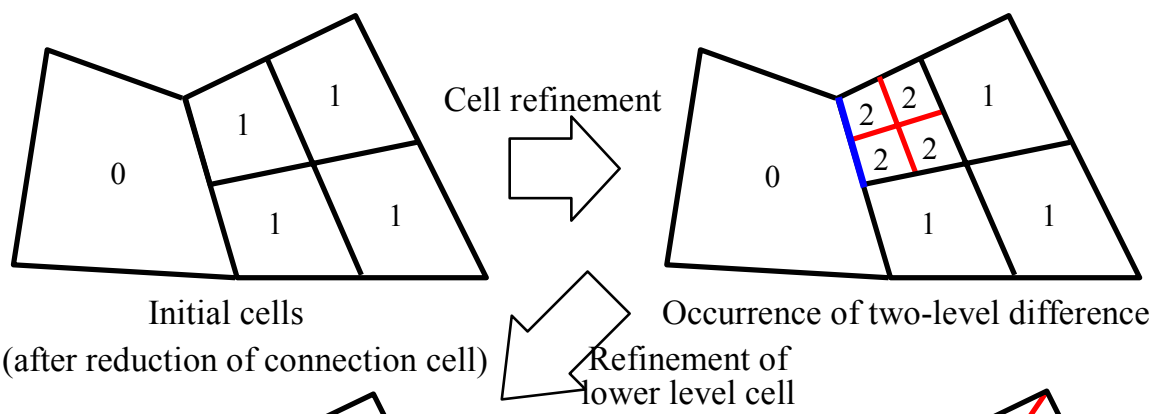

(a)
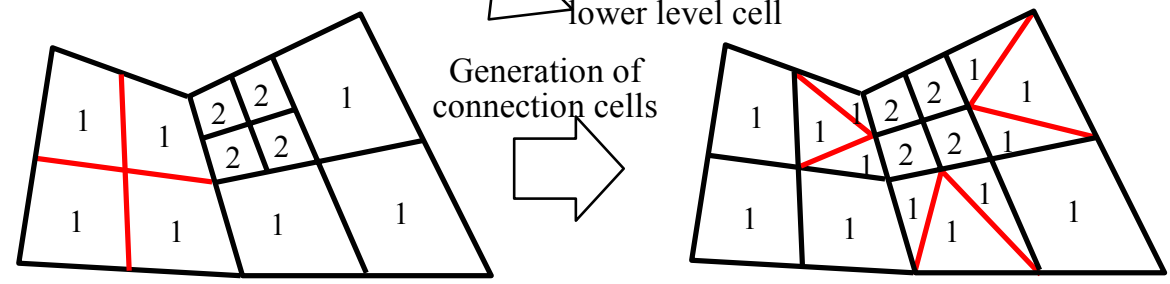

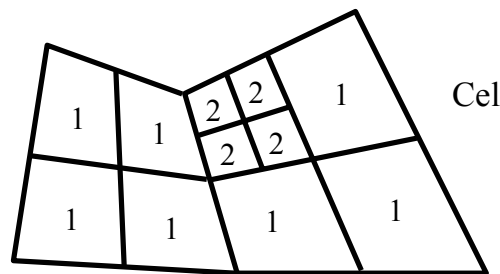

Initial cells

(after reduction of connection cell)

(b)

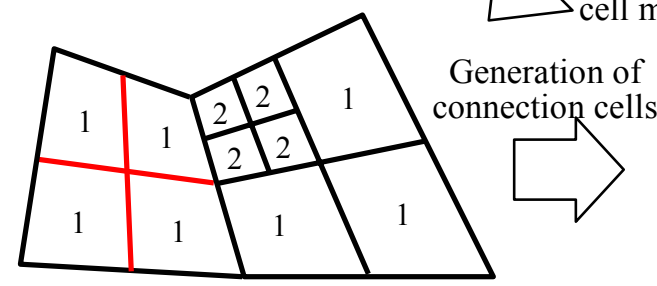

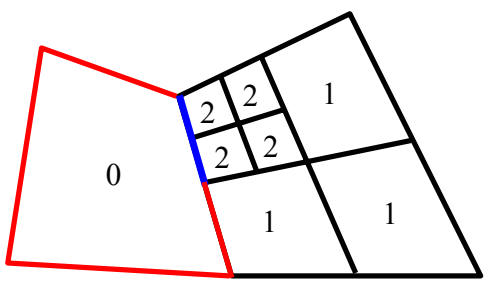

Occurrence of two-level difference Occurrence

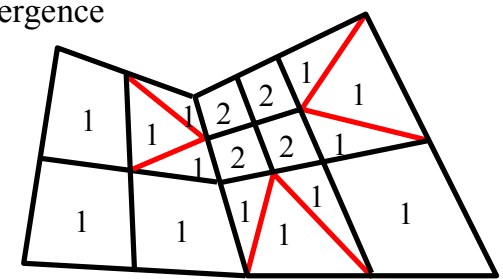

Fig. 3 Special cased for cell refinement and mergence: (a) enforced cell refinement of lower level cell, (b) cancellation of cell mergence

There is an additional rule for the refinement level. If the refinement level difference between adjacent cells becomes two (refinement level difference does not exceed two in the developed unstructured adaptive mesh technique), the cell having smaller refinement level is refined even though the indication value of the cell does not satisfy the refinement condition. This rule is effective to prevent degradation of mesh quality due to steep size change between adjacent cells and necessary to introduce connection cells properly between the adjacent cells having another refinement level. In other words, the connection cell technique is efficient to the adjacent cells having only one difference in the refinement level. Similarly, if a merged (parent) cell is adjacent to the cell having the refinement level two levels higher then the refinement level of the merged cell, this mergence is not accepted and the merged cell is refined into its child cells again. Figure 3 shows the procedures of these two special cases. Numbers in each cell show the refinement levels of the cells. In Fig. 3(a), when the two-level difference (level two and zero) between adjacent cells occurs by the refinement operation, this inadequacy is eliminated by the refinement of the cell having the lower refinement level (level zero to one). Similarly, in Fig. 3(b), two-levels difference between merged cell and adjacent small cells (level zero and two, respectively) is corrected by canceling the mergence operation and restoring the merged cell to its child cells. 


\section{Verification and Validation}

To verify and validate the two-dimensional unstructured adaptive mesh technique, the famous lid-drive square cavity flow problem was solved. Figure 4 shows the simulation conditions. A $1.0 \times 1.0$ square cavity filled with single phase fluid is surrounded by non-slip walls. Vortical flow is induced by the upper moving lid on which 1.0 horizontal (right-direction) velocity is enforced. Reynolds number is defined by the moving lid velocity (1.0), the cavity size (1.0) and the dynamic viscosity of the fluid. In this study, the authors conducted the numerical simulation under the condition where Reynolds number is 1,000 . The unsteady numerical simulation was conducted until a convergent solution was obtained. As for simulation conditions of unstructured adaptive mesh operations, velocity gradients magnitudes at each cell were employed as the indication values to set refinement and mergence flags. Namely, the larger the velocity gradient and/or magnitude at a cell, the more the cell was refined. The maximum refinement level is three.

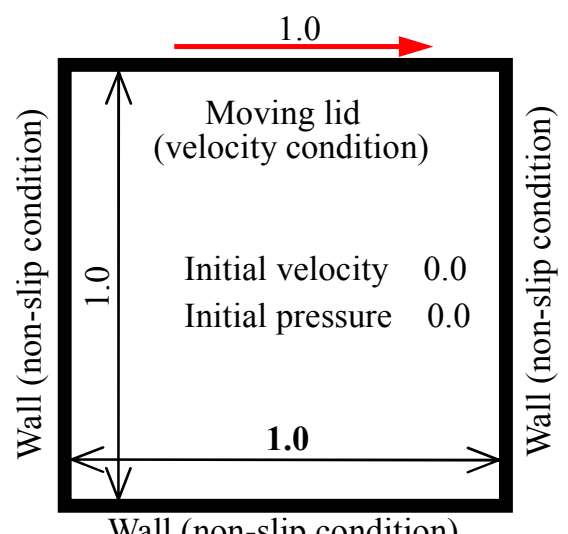

Fig. 4 Simulation conditions of lid-drive square cavity problem

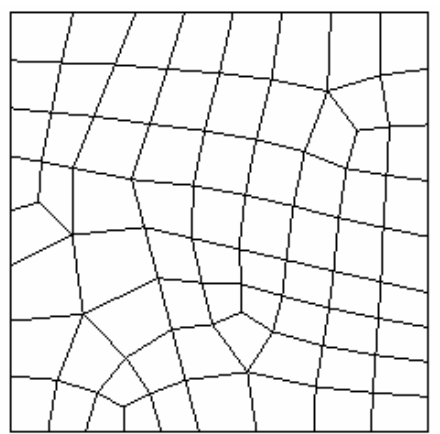

Fig. 5 Initial mesh arrangement

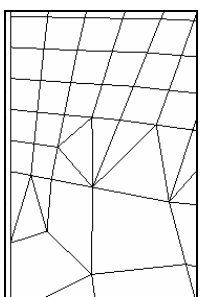

$t=2.0 \mathrm{~s}$

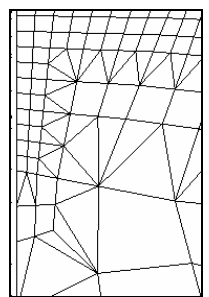

$t=4.0 \mathrm{~s}$

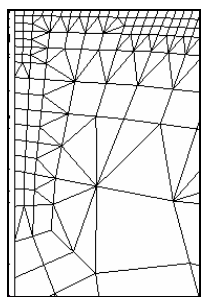

$t=6.0 \mathrm{~s}$

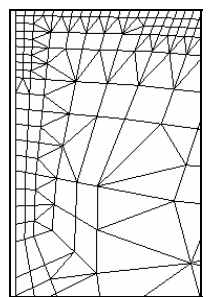

$t=8.0 \mathrm{~s}$

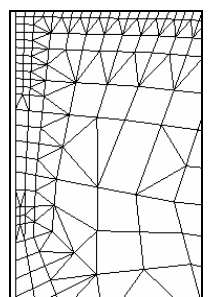

$t=10 \mathrm{~s}$

Fig. 6 Transient mesh arrangement near left wall of square cavity 
Figure 5 shows the initial mesh arrangement consisting of 71 quadrilateral unstructured cells. The authors intentionally generated the highly-irregular mesh arrangement to show that an accurate simulation result is obtained by utilizing the two-dimensional unstructured adaptive mesh technique even on such a low quality initial mesh. During the unsteady simulation, the authors checked the mesh arrangement at each time step to verify that connection cells are generated properly to eliminate the edge incompatibility between refined and non-refined cells. Figure 6 shows the transient mesh arrangement near the left wall. It is evident that the connection cells are generated around the refined cells, and the edge incompatibility is eliminated by the connection cells.

The mesh arrangement and velocity vector distribution in the square cavity at the terminal steady state are shown in Fig. 7. The terminal mesh consists of 2,931 mixed quadrilateral and triangular cells. Compared to the initial mesh (Fig. 5), the cells at large velocity gradient and magnitude regions, i.e. regions near upper moving lid and right wall, are highly refined. This improved resolution enables reproducing accurately steep velocity gradients at those regions. On the other hand, at low velocity gradient and magnitude regions, i.e. regions near the center of the vortical flow and lower two corners, the cells are coarser than the refined cell regions. Figure 8 shows the velocity distribution at the terminal steady state along the horizontal ( $x$ direction) and vertical ( $y$ direction) center lines of the square cavity. The blue plots show the simulation result on the initial mesh. This simulation result does not reproduce velocity distributions near walls and is highly inaccurate compared to the simulation result obtained on the fine structured mesh with $256 \times 256$ square cells $^{(25)}$ (shown by black solid lines). On the other hand, the simulation result on the unstructured adaptive mesh (the red plots) give very good agreement with the fine mesh result. Then, the authors also compare the simulation result on the unstructured adaptive mesh to the simulation results on structured meshes. Figure 9 shows the velocity distributions near the right wall. In this figure, the simulation results on four structured meshes $(8 \times 8,20 \times 20,40 \times 40$ and $60 \times 60)$ and two unstructured meshes (initial and adaptive) are compared. It is evident that the simulation results on the $8 \times 8$ structured and unstructured initial meshes are quite insufficient to reproduce the velocity distribution of the vortical flow. By improving the resolution to the $20 \times 20$ and $40 \times 40$ structured meshes, the velocity distribution can be simulated more accurately. Finally, the $60 \times 60$ structured and unstructured adaptive meshes make it possible to reproduce the velocity distribution. As the further comparison, the numerical error is evaluated by the comparison of each simulation result with the fine mesh result. The numerical error is calculated as:

$$
\text { Error }=\frac{\sum_{1}^{N}\left|v_{y}^{\text {sim }}-v_{y}^{\text {fine }}\right|}{N}
$$

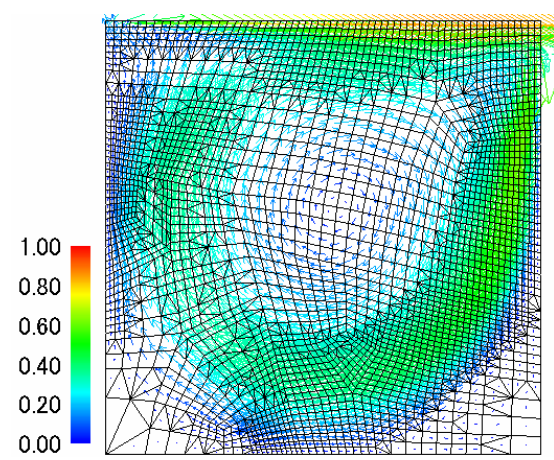

Fig. 7 Mesh arrangement and velocity distribution at terminal steady state 
Table 1 Numerical errors in lid-driven cavity flow problem

\begin{tabular}{rccc}
\hline \multicolumn{2}{c}{ Mesh } & Number of cells & Numerical error \\
\hline Structured $\quad 8 \times 8$ & 64 & $6.45 \times 10^{-2}$ \\
$20 \times 20$ & 400 & $6.18 \times 10^{-2}$ \\
$40 \times 40$ & 1,600 & $2.44 \times 10^{-2}$ \\
$60 \times 60$ & 3,600 & $1.85 \times 10^{-2}$ \\
Unstructured $\quad$ Initial & 71 & $7.04 \times 10^{-2}$ \\
& Adaptive & 2,931 & $1.35 \times 10^{-2}$ \\
\hline
\end{tabular}

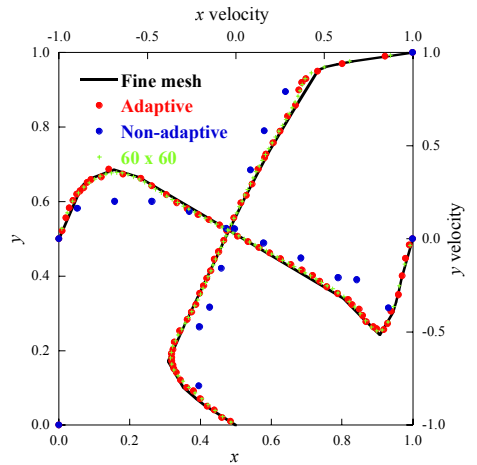

Fig. 8 Horizontal and vertical velocity distribution of vortical flow in square cavity

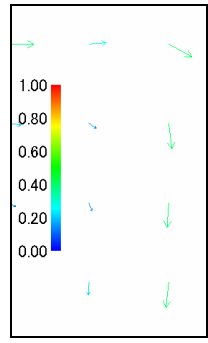

$8 \times 8$

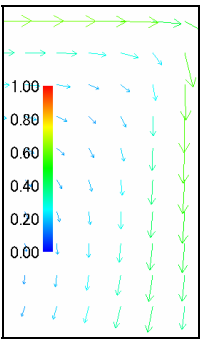

$20 \times 20$

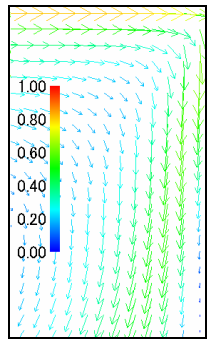

$40 \times 40$

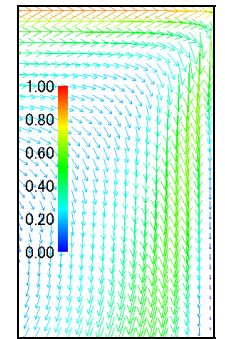

$60 \times 60$

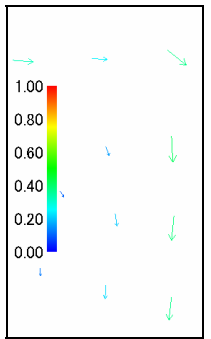

Initial

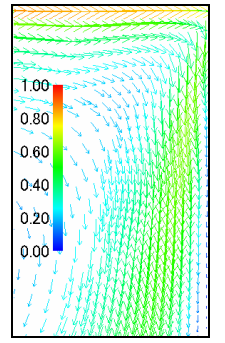

Adaptive

Structured meshes

Fig. 9 Transient mesh arrangement near left wall of square cavity

where $v_{y}$ is the vertical velocity along the horizontal center line, and $N$ is the number of cells on the horizontal center line in each simulation. The super scripts sim and fine show the simulation results in this study and the reference results on the structured fine mesh, respectively. Table 1 shows the numerical error calculated by Eq. (3). The numerical error in the simulation on the unstructured initial mesh is larger than that on the structured mesh with less number of cells $(8 \times 8)$. However, the simulation result on the unstructured adaptive mesh is more accurate than that on the structured mesh with larger number of cells $(60 \times 60)$. Therefore, it is confirmed that the unstructured adaptive mesh technique is very efficient to improve simulation accuracies for vortical flows by applying necessary cell refinements to regions where steep velocity gradients exist. It is also confirmed that this superior characteristic of the unstructured adaptive mesh technique enables to simulate vortical flows with a relatively small number of cells. In addition, the computational time consumed for the unstructured adaptive mesh operations is less than $1 / 5$ of the total computational time for one time-marching (mainly consumed for iterative pressure calculation by the BiCG-Stab algorithm). Considering the efficiency of the unstructured adaptive mesh technique, such additional computational time seems small enough. 
Furthermore, when the unstructured adaptive mesh operation is conducted every fifth or tenth time-marching (this assumption is not artificial for practical simulations), the additional computational time becomes much smaller ( $1 / 25$ or $1 / 50$, respectively).

\section{Conclusion}

In this study, as a part of the development of the high-precision and efficient two-phase flow simulation algorithm, the two-dimensional unstructured adaptive mesh technique for single-phase flows was developed and verified/validated. In the two-dimensional unstructured adaptive mesh technique, the edge compatibility was established by generating the connection cells between refined and non-refined cells. This method is useful because no special treatment (algorithm) is necessary for flow simulations on every edge. In addition, the appropriate rules for the cell refinement and mergence were determined to maintain mesh quality. As the verification/validation of the two-dimensional unstructured adaptive mesh technique, the lid-driven square cavity flow problem was simulated. As a result, it was checked that the two-dimensional unstructured adaptive mesh technique works well according to the design policy. In addition, the simulation result of the square cavity showed superior performance of the unstructured adaptive mesh technique even though the low-quality mesh was employed as the initial mesh. The refined cells were generated at the high velocity gradient regions to improve greatly the simulation accuracy. Furthermore, the simulation efficiency was investigated by comparing the simulation accuracy to the result on the structured mesh and by checking the computational time consumed for the unstructured adaptive mesh operations. As a result, the simulation result on the unstructured adaptive mesh was more accurate than the result on structured mesh with larger number of cells. In addition, the necessary computational time for the unstructured adaptive mesh technique was estimated to be small enough, e.g. less than only $1 / 50$ of the total computational time for practical simulations. From these simulation results, the authors concluded that the developed two-dimensional unstructured adaptive mesh technique is quite efficient to simulate vortical flows accurately.

\section{References}

(1) Nagata, T., Early Commercialization of Fast Reactor Cycle in Japan, The 16th Pacific Basin Nuclear Conference (16PBNC), Aomori, Japan, Oct. 13-18 (2008).

(2) Maier, M. R., Onsets of liquid and gas entrainment during discharge from a stratified air-water region through two horizontal side branches with centerlines falling in an inclined plane, M. Sc. Thesis, University of Manitoba (1998).

(3) Zuber, N., Problems in Modeling of Small Break LOCA, Nuclear Regulatory Commission Report, NUREG-0724 (1980).

(4) Eguchi, Y., Yamamoto, K., Funada, T., Tanaka, N., Moriya, S., Tanimoto, K., Ogura, K., Suzuki, K. and Maekawa, I., Gas Entrainment in the IHX of Top-Entry Loop-Type LMFBR, Nuclear Engineering and Design, Vol. 146 (1984) pp. 373-381.

(5) Sakai, T., Eguchi, Y., Monji, H., Ito, K. and Ohshima, H., Proposal of Design Criteria for Gas Entrainment From Vortex Dimples Based on a Computational Fluid Dynamics Method, Heat Transfer Engineering, Vol. 29, No. 8 (2008) pp. 731-739.

(6) Burgers, J. M., A mathematical model illustrating the theory of turbulence, Advance in applied mechanics (edited by Mises, R. and Karman, T.), Academic Press ING., New York (1948).

(7) Kimura, N., Ezure, T., Tobita, A. And Kamide, H., Experimental Study on Gas Entrainment at Free Surface in Reactor Vessel of a Compact Sodium-Cooled Fast Reactor, Journal of Nuclear Science and Technology, Vol. 45, No. 10 (2008) pp. 1053-1062. 
(8) Ito, K., Sakai, T. and Ohshima, H., Study on Applicability of Numerical Simulation to Evaluation of Gas Entrainment from Free Surface, Fourteenth International Conference on Nuclear Engineering (ICONE-14), ICONE14-89458, Miami, FL, Jul. 17-20 (2006).

(9) Okamoto, K., Takeyama, K. and Iida, M., Dynamic PIV Measurement for the Transient Behavior of a Free-Surface Vortex, Forth Japan-Korea Symposium on Nuclear Thermal Hydraulics and Safety (NTHAS4), NTHAS4-097, Sapporo, Japan (2004).

(10) Ito, K., Kunugi, T., Ohshima, H. and Kawamura, T., Formulations and Validations of a High-precision Volume-of-fluid Algorithm on Non-orthogonal Meshes for Numerical Simulations of Gas Entrainment Phenomena, Journal of Nuclear Science and Technology, Vol. 46, No. 4 (2009) pp. 366-373.

(11) Young, D. L., Time-dependent multi-material flow with large fluid distortion, in Numerical Methods for Fluid Dynamics, edited by K. W. Morton and M. J. Baines, Academic Press, New York (1982) pp. 273-468.

(12) Ito, K., Yamamoto, Y. and Kunugi, T., Development of Numerical Method for Simulation of Gas Entrainment Phenomena, The Twelfth International Topical Meeting on Nuclear Reactor Thermal Hydraulics (NURETH-12), No. 121, Pittsburgh, PA, Sep. 30 - Oct. 5 (2007).

(13) Ito, K. and Kunugi, T., Appropriate Formulations for Velocity and Pressure Calculations at Gas-liquid Interface with Collocated Variable Arrangement, Journal of Fluid Science and Technology, Vol. 4, No. 3 (2009) pp. 711-722.

(14) Takahashi, K. and Tanahashi, T., Numerical analysis of unsteady shock waves using unstructured adaptive mesh refinement (Development of scheme for sonic flows with shape flexibility and efficiency of computer memory), Transactions of the Japan Society of Mechanical Engineers, Series B, Vol. 67, No. 664 (2001), pp. 2946-2954.

(15) Vassberg, J. C., A fast, implicit unstructured-mesh Euler method, AIAA 10th Applied Aerodynamics Conference, Palo Alto, CA, June 22-24 (1992).

(16) Silva, L. F. F., Azevedo, J. L. F. and Korzenowski, H., Unstructured adaptive grid flow simulations of inert and reactive gas mixtures, J. Comput. Phys., Vol. 160 (2000) pp. $522-540$.

(17) Nakahashi, K., Ito, Y. and Togashi, F., Some challenges of realistic flow simulations by unstructured grid CFD”, Int. J. Numer. Meth. Fluids, Vol. 43 (2003) pp. 769-783.

(18) Murayama, M., Ito, Y., Nakahashi, K., Matsushima, K. and Iwamiya, T., Viscous flow computations of aircraft with changing control surface deflection using unstructured dynamic meshes, Int. J. Numer. Meth. Fluids, Vol. 52 (2006) pp. 925-940.

(19) Mavriplis, D. J., Unstructured grid technique, Annu. Rev. Fluid. Mech., Vol. 29 (1997) pp. 473-514

(20) Mavriplis, D. J., Adaptive meshing techniques for viscous flow calculations on mixed element unstructured meshes, Int. J. Numer. Meth. Fluids, Vol. 34 (2000) pp. 93-111.

(21) Kallinderis, Y. and Kavouklis, C., A daynamic adaptation scheme for general 3-D hybrid meshes, Comput. Methods Appl. Mech. Engrg., Vol. 194 (2005) pp. 5019-5050.

(22) Biswas, R. and Strawn, R. C., Tetrahedral and hexahedral mesh adaptation for CFD problems, Applied Numerical Mathematics, Vol. 26 (1998), pp. 135-151.

(23) Holmes, D. G. and Connelll, S. D., Solution of the 2D Navier-Stokes equations on unstructured adaptive grids, AIAA paper, AIAA-89-1932-CP (1989) pp. 25-39.

(24) Ito, K., Kunugi, T. and Ohshim, H., Development and Validation of Unstructured Adaptive Mesh Technique for Gas-liquid Two-phase Flows, The Thirteenth International Topical Meeting on Nuclear Reactor Thermal Hydraulics (NURETH-13), No. N13P1316, Kanazawa, Japan, Sep. 27 - Oct. 2 (2009).

(25) GHIA, U., GHIA, K. N. and SHIN, C. T., High-Re Solutions for Incompressible Flow Using the Navier-Stokes Equations and a Multigrid Method, J. Comput. Phys., Vol. 48 (1982) pp. 387-411. 